\title{
Contribution of longitudinal stiffener rigidity and position to bridge girder integrity
}

\author{
Emanuele Maiorana \\ OMBA Impianti \& Engineering SpA, Via della Croce, 10 - 36040 Torri di Quartesolo (VI), Italy \\ emaior@libero.it,bttp://orcid.org/0000-0002-3574-1410
}

\begin{abstract}
To increase the elastic critical load of a plate, such as I-shaped cross-welded section of bridge girders and upgrade bending and torsional stiffnesses, slenderness is usually reduced by dividing the web into subpanels, by means of transversal stiffeners and a longitudinal stiffener. The optimal solution is defined when the stiffener maximizes the buckling coefficient, with a minimal cross-section area. For this purpose, seven shapes of open and closed sections of longitudinal stiffeners, with different second moment of area, are examined in terms of buckling coefficients by theoretical solution and numerical computation, to compare their contribution in terms of weight per linear meter of beam. The optimum value of flexural stiffness is defined and a useful practical law is given to correlate the best position of a conventional flat stiffener with respect to variations of the stress gradient, from pure bending to pure compression, to maximize the benefits of its action and increase the stability of bridge girders.
\end{abstract}

KEYwORDS. Bridge stability; Buckling coefficient; Optimal stiffness; Stiffener optimal positioning.

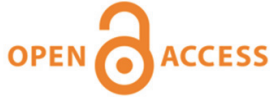

Citation: Maiorana, E., Contribution of longitudinal stiffener rigidity and position to bridge girder integrity, Frattura ed Integrità Strutturale, 48 (2019) 459-472.

Received: 20.11 .2018

Accepted: 28.02 .2019

Published: 01.04.2019

Copyright: (C) 2019 This is an open access article under the terms of the CC-BY 4.0, which permits unrestricted use, distribution, and reproduction in any medium, provided the original author and source are credited.

\section{INTRODUCTION}

$\mathrm{S}$ lender elements are widely used in engineering, as the static inertial characteristics of their sections allow full use of materials by optimization of geometric shapes according also to the external loads to which they are subjected. Generally, to increase the elastic critical load, see e.g. the bridge girders in Fig. 1, slenderness $\lambda=h / t$, i.e. the ratio between height $h$ and thickness $t$ of the web panel, is reduced by dividing the web into subpanels according to its stiffening system.

A major difficulty encountered in the study of stiffened bridge webs subject to non-uniform compression, e.g., high beam web panels subject to bending-compressive loads, is to define a sufficient rigidity and accurate positioning of the longitudinal stiffener, since compression stresses vary linearly along the web.

An optimal solution is represented by the solution in which the stiffener maximizes its effect for good web panel resistance at stability, with a minimal cross-section area. 
Although the analytical closed-form solution to determine buckling coefficient is only possible in a few simple cases of geometry and symmetry in loading (Timoshenko and Woinowsky-Krieger 1959 [1]), the Finite Element Method can be used to study many kinds of geometries, boundary conditions, and different configurations of loads, such as patch loading or local symmetrical loads, improving initial imperfections and covering a wide range of constitutive laws for materials in the non-linear range.

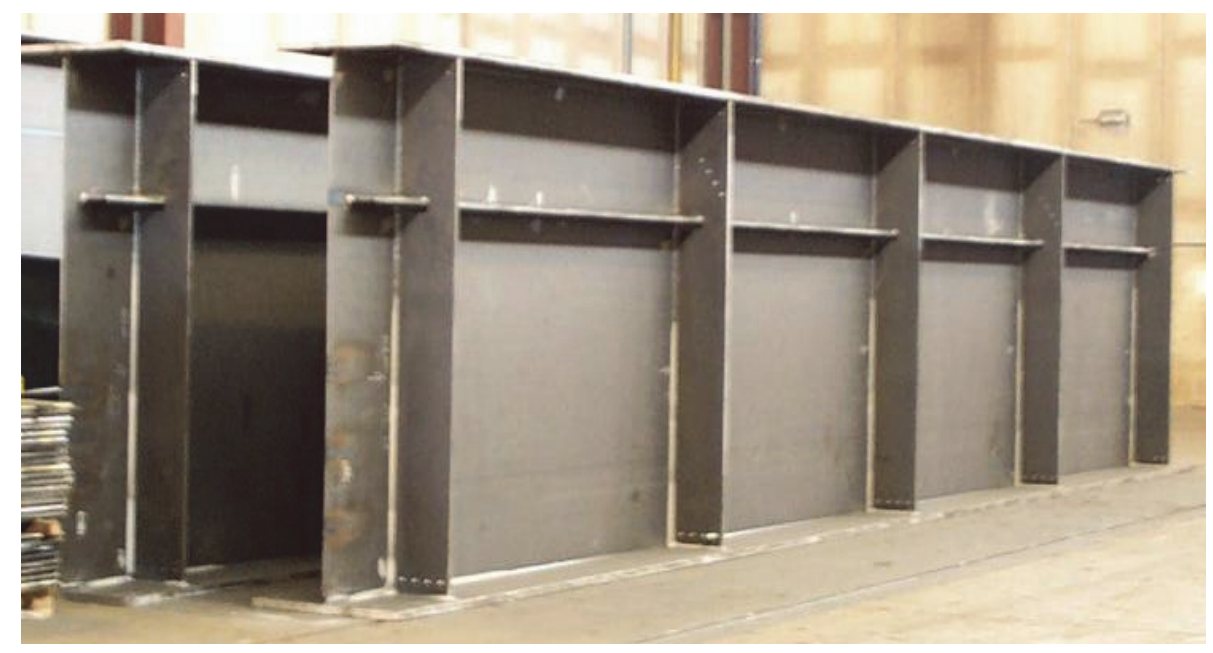

Figure 1: Bridge web panel girders strengthened by a longitudinal stiffener.

The increase in the elastic critical load through optimization of the stiffeners, by means of their shapes and positions, is examined in Xie and Chapman 2004 [2] and Lee et al. 2002 [3] and that in plates subjected to shear loading in Alinia 2005 [4]. The stability of longitudinally stiffened web plates under interactive shear and bending force is described in Alinia and Moosavi 2009 [5] and the influence of longitudinal stiffeners on the elastic stability of girder webs in Maiorana et al. 2011 [6]. An improved design procedure for efficient design of stiffened plates, under combined compression and bending, and in detail the influence of the stiffener location on the stability of the structure, finding the optimum location of it, is proposed in Bedair, 1997 [7].

The ultimate strength of longitudinally stiffened I-girder webs, subjected to combined patch loading and bending, is treated in Graciano and Casanova 2005 [8] and the shear strength of longitudinally stiffened panels with imperfections in Pavlovčič et al. 2007 [9].

The beams examined in this work are reinforced with a longitudinal stiffener and some generalizations were made to improve stiffener design with regard to the rigidity and position, in order to maximize elastic stability.

The longitudinal stiffener was connected to the plate at various positions $b^{\prime} / b$ with respect to the compressed edge, so that the panel of length $a$ at height $h$ was divided into two subpanel: upper plate A with height $b^{\prime}$, and the lower plate B with height $h^{\prime \prime}$.

A Finite Element model was used according to the Strand7 code (G+D Computing 2005 [10]), with a static scheme consisting of a simple hinge bounded plate subjected to combination of bending moment and compression forces (Fig. 2). A sensitivity analysis of the mesh, to define the shape and dimension of the elements was treated deeply in previous papers by the author of this paper. Maiorana and Pellegrino 2011 [11] compares k values obtained with the FE model and Eurocode equations for square and rectangular panels of constant thickness subjected to axial force and bending moment, and Pellegrino et al. 2009 [12] compares the results of the FE model with those of the literature, in the case of perforated steel plates under shear loading.

The FE used for the mesh were plates with four nodes (Quad4) and six degrees of freedom per node; the typical size of the elements being $h / 20$.

Boundary conditions were assumed to represent the plate as a web panel, see Fig. 2: sides 1, 2, 3 and 4 displacements restrain $u_{\mathrm{y}}=0$; side 2 and $4 u_{\mathrm{z}}=0$; and displacements $u_{\mathrm{x}}$ were symmetric.

The applied loads were in equilibrium and directly applied to the nodes on the two lateral vertical edges as a system of conservative forces which do not change direction during deformation, due to symmetry. 


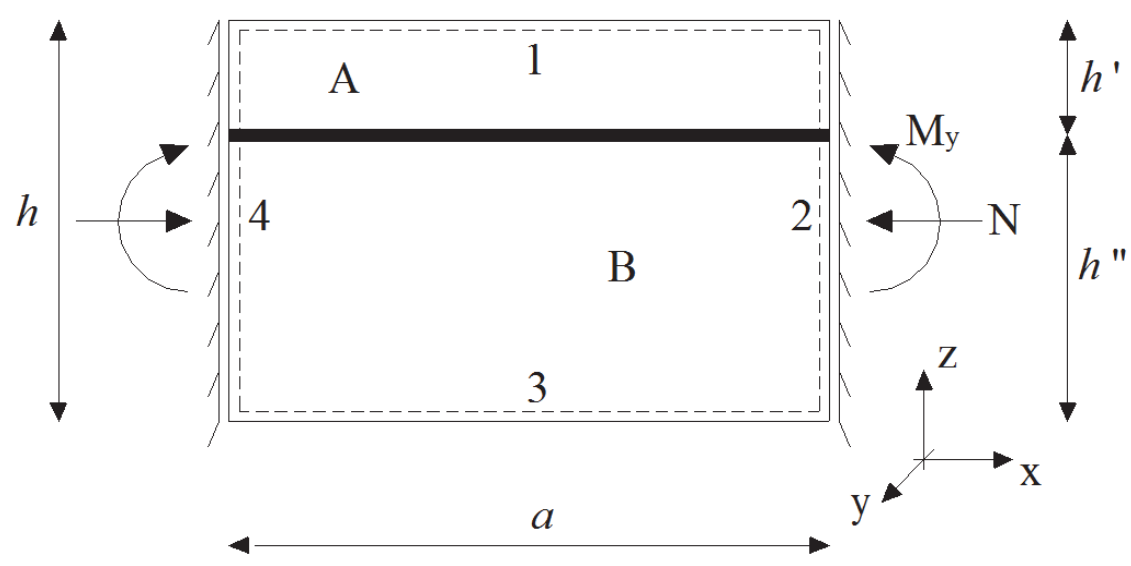

Figure 2: Static diagram of a plate divided into two subpanels A and B; $a / b=1.5$.

\section{BASIC CONCEPT}

\section{Buckling coefficient}

7 heoretical buckling coefficients $k_{\text {th }}$ of simply supported plates subjected to uniform compression were analytically determined by solving the Timoshenko's equations based on equilibrium between external and internal forces, in a deformed configuration.

The lower value of the compression force $N_{\mathrm{x}}$ is obtained with $n=1$, and the buckling coefficient is given by Eqn. 1:

$$
k_{\text {th }}=[(m b) / a+a /(m b)]^{2}
$$

Being stress gradient $\psi=\sigma_{\mathrm{t}} / \sigma_{\mathrm{c}}$ the ratio between traction $\sigma_{\mathrm{t}}$ and compression $\sigma_{\mathrm{c}}$ stresses, in EN 1993-1-5 2007 [13] the following $k$ values are proposed:

$$
\begin{array}{ll}
k=4 & \text { for } \psi=1 \\
k=8.2 /(1.05+\psi) & \text { for } 1>\psi>0 \\
k=7.81 & \text { for } \psi=0 \\
k=7.81-6.29 \psi+9.7 \psi^{2} & \text { for } 0>\psi>-1 \\
k=23.9 & \text { for } \psi=-1 \\
k=5.98\left(1-\psi^{2}\right) & \text { for }-1>\psi>-3
\end{array}
$$

Numerically, buckling coefficient $k_{\text {num }}$ was found by solving the corresponding eigenvalue problem. The lower eigenvalue refers to the critical elastic load and the eigenvector defines its deformed shape.

Stiffness matrix $\boldsymbol{K}$ was given by the conventional matrix in small deformations $\boldsymbol{K}_{\mathrm{E}}$ and the matrix $\boldsymbol{K}_{\mathrm{S}}$, which takes into account the effect of stress $\sigma$ on the plate. The global stiffness matrix of the panel at stress level $\sigma_{0}$ may be written as follows:

$$
\boldsymbol{K}\left(\sigma_{0}\right)=\boldsymbol{K}_{\mathrm{E}}+\boldsymbol{K}_{\mathrm{S}}\left(\sigma_{0}\right)
$$

When the stress level reaches $\lambda \sigma_{0}$, the stiffness matrix becomes: 


$$
\boldsymbol{K}\left(\lambda \sigma_{0}\right)=\boldsymbol{K}_{\mathrm{E}}+\boldsymbol{K}_{\mathrm{S}}\left(\lambda \sigma_{0}\right)
$$

The equation governing plate behavior is:

$$
d \boldsymbol{F}=\left[\boldsymbol{K}_{\mathrm{E}}+\lambda \boldsymbol{K}_{\mathrm{S}}\left(\sigma_{0}\right)\right] d \boldsymbol{u}
$$

where $d \boldsymbol{u}$ is the vector of displacement variations and $d \boldsymbol{F}$ the vector of load variations. The determinant of the matrix becomes null at buckling, and an increase in displacement without a corresponding increase in load occurs:

$$
\left[\boldsymbol{K}_{\mathrm{E}}+\lambda \boldsymbol{K}_{\mathrm{S}}\left(\sigma_{0}\right)\right] d \boldsymbol{u}=0
$$

The solution to this is an eigenvalue problem corresponding to the lower eigenvalue $\lambda_{1}$ related to critical elastic stress, at which buckling occurs:

$$
\sigma_{\mathrm{cr}}=\lambda_{1} \sigma_{0}
$$

\section{Optimal stiffness and best position}

On the base of relative bending stiffness, the behavior of the whole web panel-stiffener system can be divided into two categories, defined by the different mechanism of the loss of stability. The flexible stiffening system is characterized by the fact that the web panel and the stiffener reach instability together in a global way, whereas the rigid stiffening system has a local instability mode.

In the first case, both web panel and stiffening have the same elastic critical load and the optimal position changes and must be determined numerically for a given relative flexural stiffness ratio for every value of Eqn. 7 (Dubas and Gehri 1986 [14]).

$$
\gamma_{\mathrm{s}}=\left(E I_{\mathrm{st}}\right) /(D h)
$$

where $D$ in the flexural rigidity by Eqn. 8

$$
D=\left(E t^{3}\right) /\left[12\left(1-v^{2}\right)\right]
$$

In the second case, stiffening is sufficient against any loss of stability of the web panel, forming a nodal line, and the overall buckling load is the lower value, between that of the subpanels A and B.

Both deformation modes, flexible and rigid, occur together and interact closely in the typical buckling shapes of plates with semi-rigid stiffeners, classified as a transient stage. Reaching optimal stiffness, these buckling shapes do not interact (Alinia 2005 [4]) and stiffening remains straight until complete deformation has taken place.

The optimal position of the stiffener is reached when the elastic critical load is the same in both subpanels and also depends on stress gradient $\psi$ : for $\psi<0$ bending dominates compression forces, whereas for $\psi>0$ compression dominates the bending moment. Thus, for a given value of stress gradient $\psi$, the distribution of forces changes in subpanels A and B (Fig. 1) and, accordingly, the optimal position changes. Increasing $\psi$, the optimal position of the stiffener moves to the centerline of the plate, regardless of relative stiffness (Bedair 1997 [7]).

In the design of open beams subject to pure bending forces, the American code (AASHTO 2018 [15]) recommends the longitudinal stiffener to be positioned at an average distance of $h^{\prime} / b=0.2$ from the compressed edge. This position depends on the ratio between rigidities of stiffener and plate, and is not always exactly the same for each situation but changes according to relative flexural stiffness $\gamma_{\mathrm{s}}$. In fact, unlike the case of prevailing compression stresses, when the bending moment predominates, the optimal location for a longitudinal stiffener depends on the geometrical characteristics of the whole plate/stiffener relationship. In conclusion, reaching optimal stiffness with stiffener in its optimum position, maximal stability is reached, ensuring that the stiffener has a minimal cross-sectional area.

\section{Effect of initial imperfection}

Many national steel construction codes prescribe the maximum size/magnitude of the initial imperfection relating to panel height and width. Generally standards take into account the influence of thickness, or refer to slenderness. European Standard (EN 1090-2 2018 [16]) refers to the slenderness, directly with $b / t$ (local) or indirectly with $L / 300$ (global). 
Prescriptions are developed and used without any reference to the applied loads acting on the panels in question, so that the particular contexts of application are neglected.

For the purposes of this study, the assumption is considering a perfect plane plate without out-of-plane eccentricities. The scope is to find buckling coefficients $k$ from linear eigenbuckling analysis considering the loss of stability from 1 st buckling mode.

\section{STIFFENER CROSS-SECTION SHAPE}

$\mathrm{O}$

pen-section and closed-section longitudinal stiffeners are considered, each respecting the geometric relationships of dimensions according to EN 1993-1-5 2007 [13].

For comparisons, seven cross-sections having an equal value of area but different second moments of area (Table 1), i.e. three open cross-sections and four closed ones, have been examined. The choice was dictated by the need to consider the same contribution in terms of weight per linear meter of deck beam.

Fig. 3 compares solutions of linear buckling coefficients for non-stiffened plate US0 and stiffened plates with the seven types of stiffeners, with respect to the plate aspect ratios $\alpha=a / b$ (see Fig. 2).

Cross-section CC6 appeared to be the best stiffener shape to stiffen the plate, for the usual range of web panel aspect ratios, i.e. $1.5<\alpha \leq 2$. For $\alpha<1.5$, cross-section CR5 shows a greater value than CC6.

CT4 makes the same contribution to stability varying $\alpha$. For the open-section stiffeners, the best characteristics were found in OT2 and OL3 that show higher second moments of area; OF1 makes the lowest contribution to the increase of buckling coefficient $k$. With respect to open cross-sections, in CR5, CC6 and CZ7, $k$ decreases as $\alpha$ increases; local instabilities occur in closed cross-sections with greater slenderness.

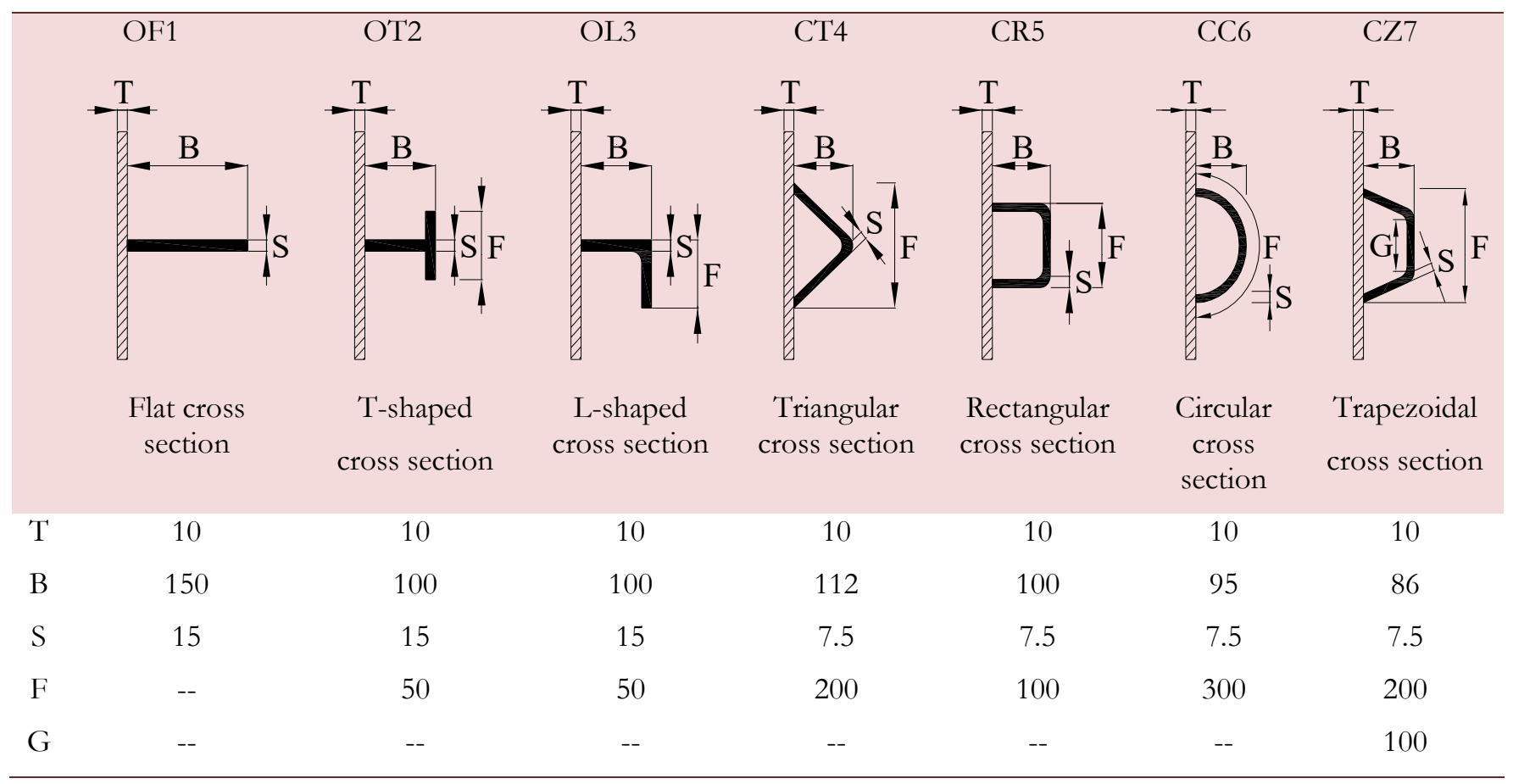

Table 1: Stiffeners: analysed cross-sectional shape and geometrical dimensions ( $\mathrm{mm})$.

Being equal the area $A_{\mathrm{st}}$, it is possible to improve stiffening performance by maximizing the second moment of area $I_{\mathrm{st}}$; a considerable increase in the linear buckling coefficient could be reached by using closed-section stiffeners rather than open types.

Fig. 4 compares solutions for the buckling coefficient of a stiffened plate with aspect ratio $\alpha=1.5$ and position of longitudinal stiffener $b^{\prime} / b=0.2$. For the stiffened plate, the minimal value of buckling coefficient $k$ is 5.73 for cross-section OF1 and 8.26 for CC6. 


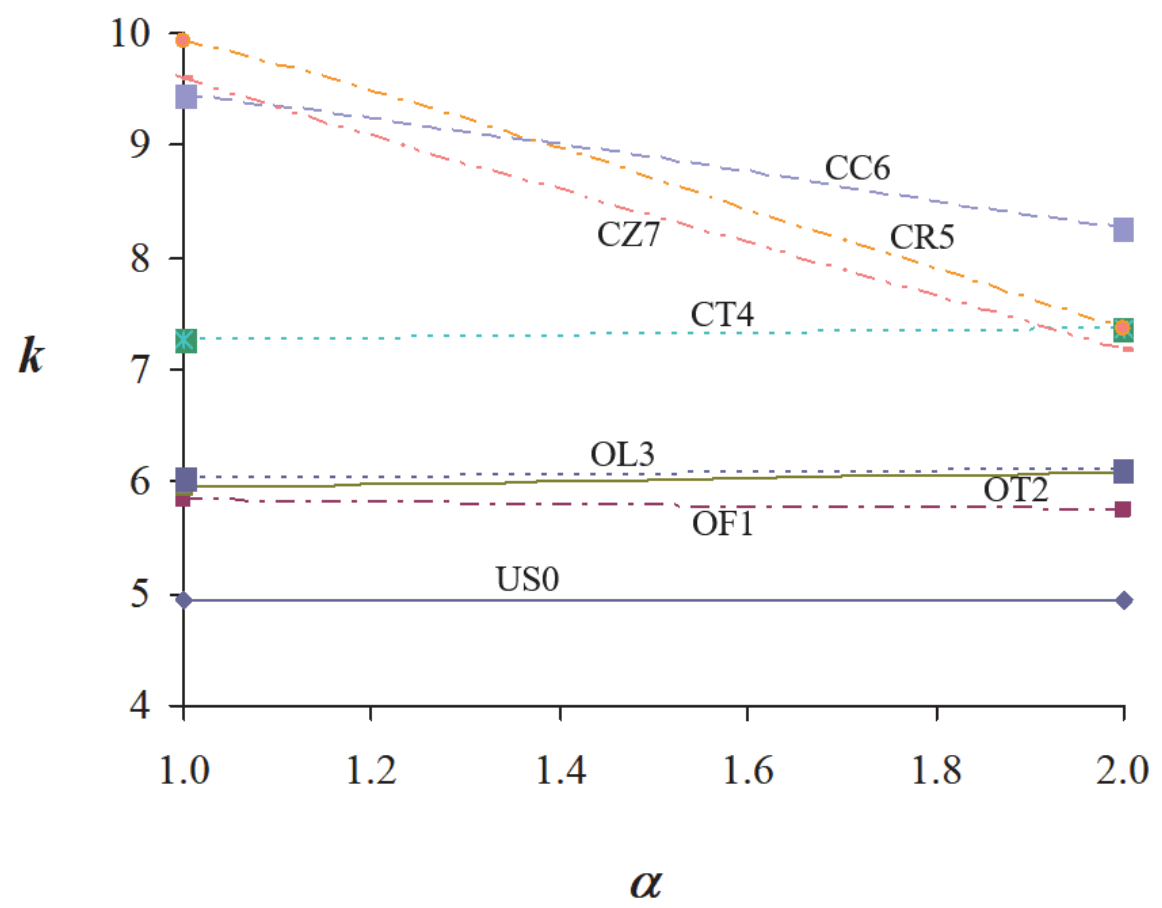

Figure 3: Buckling coefficient $k$ vs. aspect ratio $\alpha$ for plate with and without stiffener.

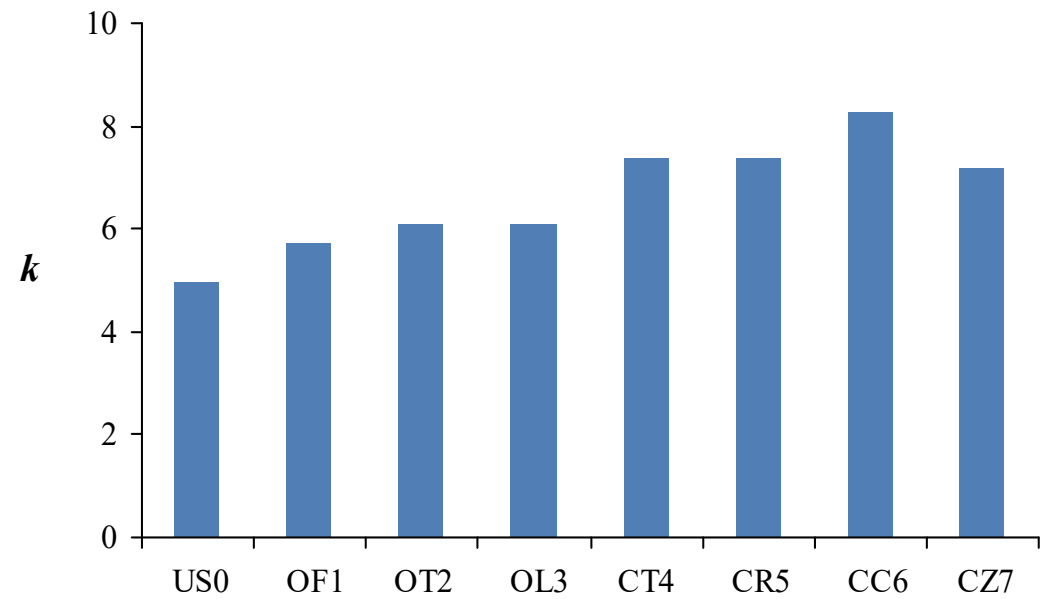

Figure 4: Comparison of solutions for $k$ in plates under bending moment $\psi=-1$ for unstiffened plate US and with differing stiffener cross-section shapes, open $\mathrm{O}$ and closed $\mathrm{C}$.

Correctly, the solution for the non-stiffened plate US0 has the lowest value of the group. In Fig. 5 and Fig. 6 a comparison of buckling coefficients according to differing cross-section shapes is proposed, varying aspect ratio $\alpha$, with range from 0.5 to 1.5 ; after $\alpha=1.5$, linear buckling coefficient values are constant.

Fig. 5 shows the solutions of $k$, comparing non-stiffened plates with open cross-section ones. Fig. 6 shows the solutions of $k$, comparing non-stiffened plates with closed cross-section ones. For $\alpha<1$, cross-section CZ7 is the best.

Tables 2-8 list the buckling coefficient values according to the theory $\left(k_{\mathrm{th}}\right)$ and derived from numerical evaluation $\left(k_{\text {num }}\right)$. The difference between theoretical $k_{\text {th }}$ and numerical $k_{\text {num }}$ values is considerably large in many cases and numerical values are greater. This may be explained in two ways: 1) the numerical results show the restraining effect of each subpanel caused by the complementary one; 2) section area being equal, the second moment of area is not considered in theoretical calculations. 


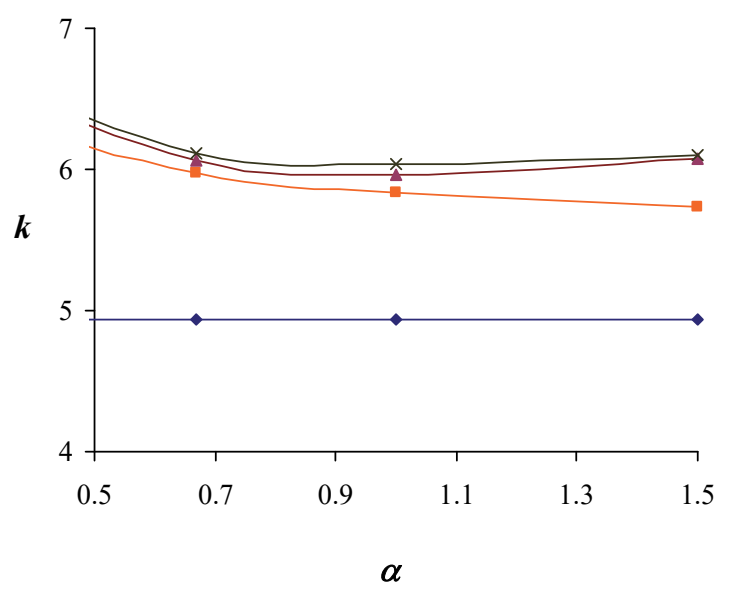

(a)

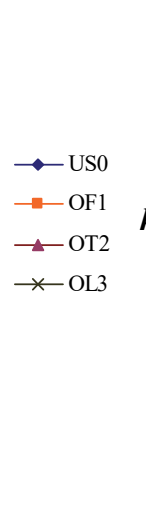

20

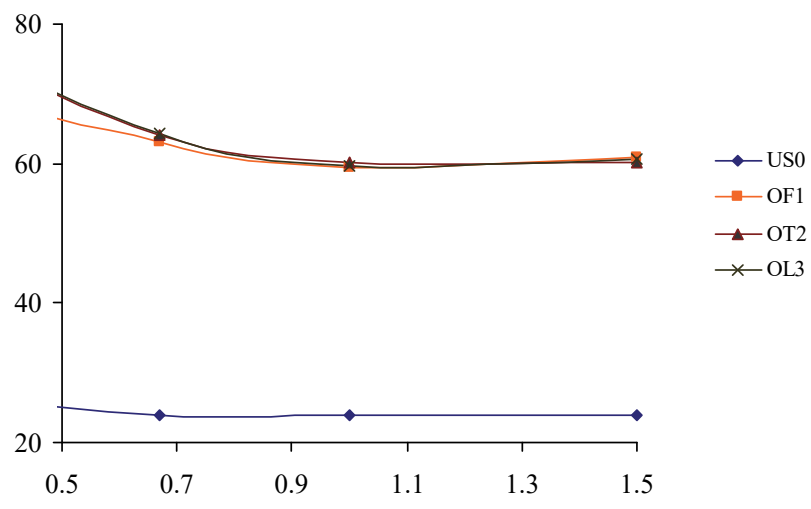

(b)

Figure 5: Buckling coefficient $k$ vs. aspect ratio $\alpha$ with open section stiffening; subpanel A (a) and B (b).

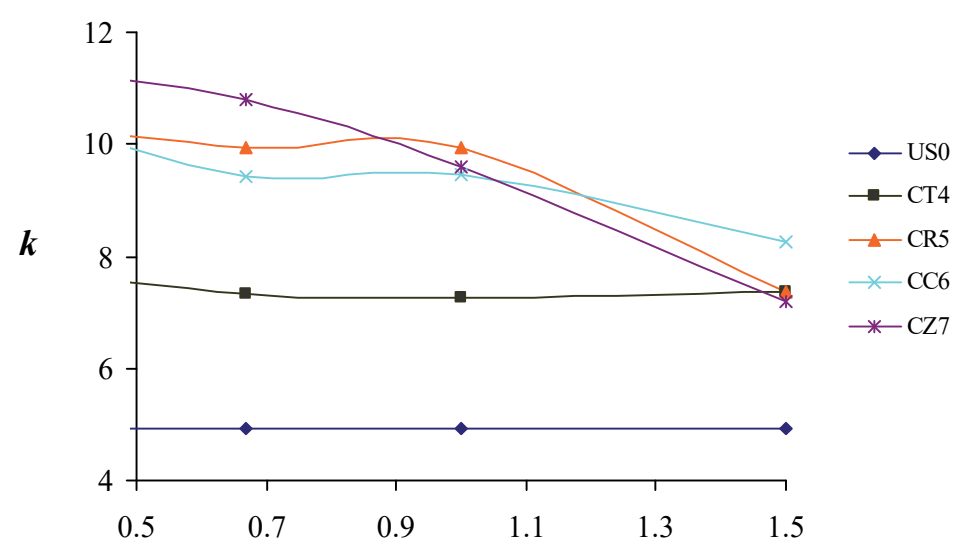

$\alpha$

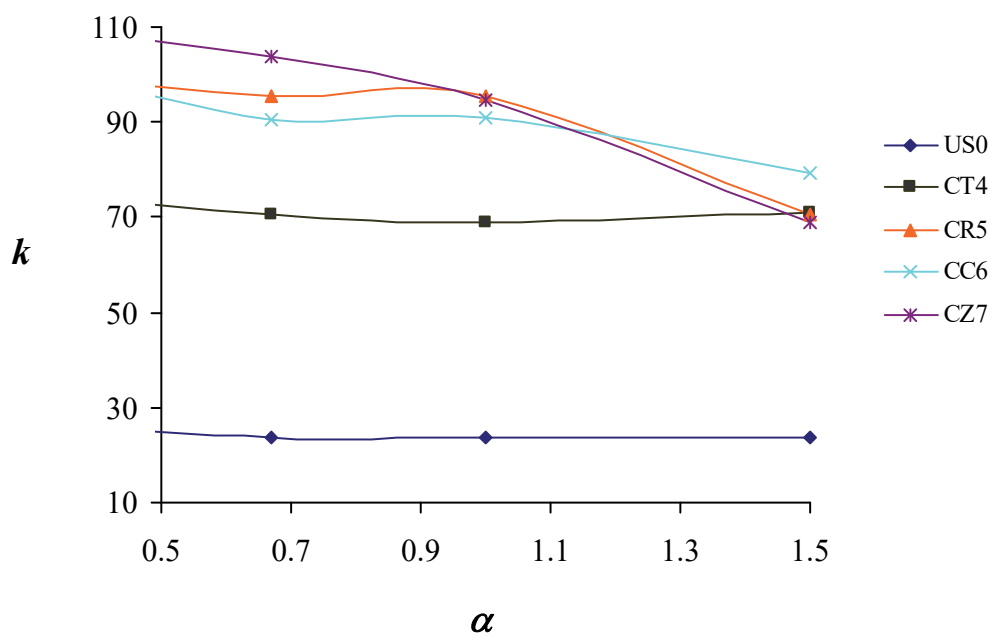

(a)

(b)

Figure 6: Buckling coefficient $k$ vs. aspect ratio $\alpha$ with close section stiffening; subpanel A (a) and B (b). 


\begin{tabular}{|c|c|c|c|c|c|c|c|}
\hline \multirow[t]{2}{*}{$\alpha$} & \multirow[t]{2}{*}{$b^{\prime} / b$} & \multicolumn{2}{|c|}{$\psi=-1$} & \multicolumn{2}{|c|}{$\psi=0$} & \multicolumn{2}{|c|}{$\psi=1$} \\
\hline & & $k_{\mathrm{th}}$ & $k_{\text {num }}$ & $k_{\text {th }}$ & $k_{\text {num }}$ & $k_{\mathrm{th}}$ & $k_{\text {num }}$ \\
\hline \multirow{4}{*}{1.50} & 0.20 & 4.94 & 5.73 & 7.63 & 9.49 & 4.00 & 5.62 \\
\hline & 0.30 & 5.60 & 6.43 & 7.63 & 9.42 & 4.00 & 5.43 \\
\hline & 0.50 & 7.60 & 8.59 & 5.25 & 5.85 & 4.00 & 4.86 \\
\hline & 0.20 & 4.94 & 5.84 & 7.63 & 9.96 & 4.00 & 6.35 \\
\hline \multirow[t]{3}{*}{1.00} & 0.30 & 5.60 & 6.63 & 7.63 & 9.18 & 4.00 & 6.19 \\
\hline & 0.50 & 7.60 & 8.97 & 5.25 & 5.80 & 4.00 & 5.15 \\
\hline & 0.20 & 4.94 & 5.98 & 7.63 & 8.85 & 4.46 & 8.70 \\
\hline \multirow[t]{2}{*}{0.67} & 0.30 & 5.60 & 6.91 & 7.63 & 8.62 & 4.33 & 7.13 \\
\hline & 0.50 & 7.60 & 10.34 & 5.25 & 6.05 & 4.00 & 5.54 \\
\hline
\end{tabular}

Table 2: Linear buckling coefficient for cross-section OF1.

\begin{tabular}{cccccccc}
\hline$\alpha$ & $b^{\prime} / h$ & \multicolumn{2}{c}{$\psi=-1$} & \multicolumn{3}{c}{$\psi=1$} \\
& & $k_{\text {th }}$ & $k_{\text {num }}$ & $k_{\text {th }}$ & $k_{\text {num }}$ & $k_{\text {th }}$ & $k_{\text {num }}$ \\
\multirow{4}{*}{1.50} & 0.20 & 4.94 & 6.08 & 7.63 & 9.13 & 4.00 & 5.62 \\
& 0.30 & 5.60 & 6.51 & 7.63 & 9.05 & 4.00 & 5.42 \\
& 0.50 & 7.60 & 8.61 & 5.25 & 5.94 & 4.00 & 4.62 \\
\multirow{4}{*}{0.00} & 0.20 & 4.94 & 5.96 & 7.63 & 9.74 & 4.00 & 6.35 \\
& 0.30 & 5.60 & 6.67 & 7.63 & 8.90 & 4.00 & 6.18 \\
& 0.50 & 7.60 & 8.97 & 5.25 & 5.85 & 4.00 & 5.25 \\
& 0.20 & 4.94 & 6.06 & 7.63 & 8.60 & 4.46 & 8.59 \\
& 0.30 & 5.60 & 6.98 & 7.63 & 8.30 & 4.33 & 7.13 \\
\hline
\end{tabular}

Table 3: Linear buckling coefficient for cross-section OT2.

\begin{tabular}{cccccccc}
\hline$\alpha$ & $h^{\prime} / h$ & \multicolumn{2}{c}{$\psi=-1$} & & \multicolumn{2}{c}{$\psi=1$} \\
& & $k_{\text {th }}$ & $k_{\text {num }}$ & $k_{\text {th }}$ & $k_{\text {num }}$ & $k_{\text {th }}$ & $k_{\text {num }}$ \\
\multirow{4}{*}{1.50} & 0.20 & 4.94 & 6.10 & 7.63 & 8.96 & 4.00 & 5.62 \\
& 0.30 & 5.60 & 6.60 & 7.63 & 8.90 & 4.00 & 5.43 \\
& 0.50 & 7.60 & 8.54 & 5.25 & 6.05 & 4.00 & 5.02 \\
\multirow{3}{*}{0.00} & 0.20 & 4.94 & 6.04 & 7.63 & 9.67 & 4.00 & 6.27 \\
& 0.30 & 5.60 & 6.74 & 7.603 & 8.84 & 4.00 & 6.12 \\
& 0.50 & 7.60 & 9.05 & 5.25 & 5.97 & 4.00 & 5.34 \\
& 0.20 & 4.94 & 6.12 & 7.63 & 8.47 & 4.46 & 8.08 \\
& 0.30 & 5.60 & 7.11 & 7.63 & 8.18 & 4.33 & 7.13 \\
\hline
\end{tabular}

Table 4: Linear buckling coefficient for cross-section OL3. 


\begin{tabular}{ccccccccc}
\hline$\alpha$ & $b^{\prime} / h$ & \multicolumn{2}{c}{$\psi=-1$} & \multicolumn{3}{c}{$\psi=0$} & \\
& & $k_{\text {th }}$ & $k_{\text {num }}$ & $k_{\text {th }}$ & $k_{\text {num }}$ & $k_{\text {th }}$ & $k_{\text {num }}$ \\
\multirow{4}{*}{1.50} & 0.20 & 4.94 & 7.37 & 7.63 & 10.89 & 4.00 & 6.18 \\
& 0.30 & 5.60 & 7.07 & 7.63 & 10.57 & 4.00 & 5.43 \\
& 0.50 & 7.60 & 9.23 & 5.25 & 6.74 & 4.00 & 6.12 \\
& 0.20 & 4.94 & 7.27 & 7.63 & 10.89 & 4.00 & 7.09 \\
& 0.30 & 5.60 & 7.52 & 7.63 & 10.42 & 4.00 & 3.22 \\
& 0.50 & 7.60 & 10.40 & 5.25 & 6.86 & 4.00 & 6.31 \\
& 0.20 & 4.94 & 7.33 & 7.63 & 10.13 & 4.46 & 8.50 \\
& 0.30 & 5.60 & 7.93 & 7.63 & 10.16 & 4.33 & 7.13 \\
& 0.50 & 7.60 & 11.49 & 5.25 & 6.68 & 4.00 & 7.18 \\
\hline
\end{tabular}

Table 5: Linear buckling coefficient for cross-section CT4.

\begin{tabular}{cccccccc}
\hline$\alpha$ & $b^{\prime} / h$ & \multicolumn{2}{c}{$\psi=-1$} & \multicolumn{3}{c}{$\psi=0$} & \\
& & $k_{\text {th }}$ & $k_{\text {num }}$ & $k_{\text {th }}$ & $k_{\text {num }}$ & $k_{\text {th }}$ & $k_{\text {num }}$ \\
\multirow{4}{*}{1.50} & 0.20 & 4.94 & 7.36 & 7.63 & 11.56 & 4.00 & 6.51 \\
& 0.30 & 5.60 & 8.75 & 7.63 & 11.26 & 4.00 & 6.63 \\
& 0.50 & 7.60 & 9.80 & 5.25 & 7.23 & 4.00 & 6.89 \\
\multirow{3}{*}{1.00} & 0.20 & 4.94 & 9.93 & 7.63 & 11.56 & 4.00 & 7.42 \\
& 0.30 & 5.60 & 8.84 & 7.63 & 11.32 & 4.00 & 7.38 \\
& 0.50 & 7.60 & 10.30 & 5.25 & 7.25 & 4.00 & 7.28 \\
& 0.20 & 4.94 & 9.93 & 7.63 & 10.33 & 4.46 & 8.66 \\
& 0.30 & 5.60 & 9.06 & 7.63 & 10.58 & 4.33 & 7.95 \\
& 0.50 & 7.60 & 11.78 & 5.25 & 7.12 & 4.00 & 7.83 \\
\hline
\end{tabular}

Table 6: Linear buckling coefficient for cross-section CR5.

\begin{tabular}{cccccccc}
\hline$\alpha$ & $b^{\prime} / h$ & \multicolumn{2}{c}{$\psi=-1$} & \multicolumn{3}{c}{$\psi=0$} & \\
& & $k_{\text {th }}$ & $k_{\text {num }}$ & $k_{\text {th }}$ & $k_{\text {num }}$ & $k_{\text {th }}$ & $k_{\text {num }}$ \\
\multirow{4}{*}{1.50} & 0.20 & 4.94 & 8.26 & 7.63 & 11.44 & 4.00 & 6.51 \\
& 0.30 & 5.60 & 8.57 & 7.63 & 11.24 & 4.00 & 6.56 \\
& 0.50 & 7.60 & 9.80 & 5.25 & 7.21 & 4.00 & 6.63 \\
& 0.20 & 4.94 & 9.45 & 7.63 & 11.55 & 4.00 & 7.51 \\
& 0.30 & 5.60 & 8.61 & 7.63 & 11.34 & 4.00 & 7.32 \\
& 0.50 & 7.60 & 10.30 & 5.25 & 7.22 & 4.00 & 6.96 \\
& 0.20 & 4.94 & 9.43 & 7.63 & 10.25 & 4.46 & 8.66 \\
& 0.30 & 5.60 & 8.77 & 7.63 & 10.50 & 4.33 & 7.89 \\
& 0.50 & 7.60 & 11.78 & 5.25 & 7.10 & 4.00 & 7.70 \\
\hline
\end{tabular}

Table 7: Linear buckling coefficient for cross-section CC6. 


\begin{tabular}{ccccccccc}
\hline$\alpha$ & $b^{\prime} / h$ & \multicolumn{2}{c}{$\psi=-1$} & \multicolumn{3}{c}{$\psi=1$} & \\
& & $k_{\text {th }}$ & $k_{\text {num }}$ & $k_{\text {th }}$ & $k_{\text {num }}$ & $k_{\text {th }}$ & $k_{\text {num }}$ \\
\multirow{4}{*}{1.50} & 0.20 & 4.94 & 7.18 & 7.63 & 11.92 & 4.00 & 6.68 \\
& 0.30 & 5.60 & 9.19 & 7.63 & 13.22 & 4.00 & 6.75 \\
& 0.50 & 7.60 & 9.94 & 5.25 & 7.51 & 4.00 & 6.89 \\
& 0.20 & 4.94 & 9.60 & 7.63 & 11.88 & 4.00 & 7.59 \\
& 0.30 & 5.60 & 9.29 & 7.63 & 11.64 & 4.00 & 7.45 \\
& 0.50 & 7.60 & 10.55 & 5.25 & 7.46 & 4.00 & 7.12 \\
& 0.20 & 4.94 & 10.80 & 7.63 & 10.78 & 4.46 & 8.74 \\
& 0.30 & 5.60 & 9.46 & 7.63 & 11.12 & 4.33 & 8.08 \\
& 0.50 & 7.60 & 11.88 & 5.25 & 7.34 & 4.00 & 7.95 \\
\hline
\end{tabular}

Table 8: Linear buckling coefficient for cross-section CZ7.

\section{STIFFENER POSITIONING}

he optimal position of a stiffener with regard to web panel height is a function of the compressed subpanel (Fig. 7). For $\psi=1$, pure compression, the optimal position is $h^{\prime} / h=0.5$, whereas for $\psi=-1$, pure bending, is $h^{\prime} / h=$ 0.2 from the compressed edge. Linear buckling analyses has been done for various positions of a longitudinal stiffener, from $h^{\prime} / h=0.14$ to 0.5 with step 0.01 , to cover the whole area of the compressed subpanel while $\psi$ was set from -1 to 1 with step 0.25 . A conventional flat stiffener was chosen, i.e. cross-section OF1, the simplest section among those in Tab. 1.

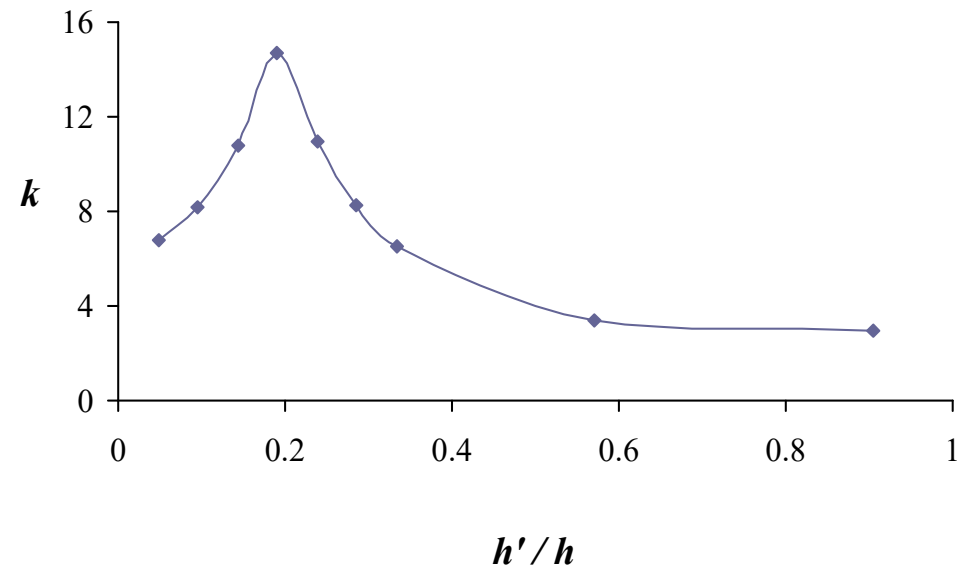

Figure 7: Buckling coefficient $k$ vs. stiffener position $h^{\prime} / h$ for plate under pure bending moment.

In order to analyse the variability of stiffeners geometric dimensions with respect to plate geometry, i.e., relative flexural stiffness, the relationship between stiffener thickness and width versus the elastic critical load of the plate was established. The resulting thickness/width groups were identified and four types were found (Fig. 8).

In case OF1-I, the typical dimensions of the longitudinal stiffener were $b_{\mathrm{st}}=300 \mathrm{~mm}$ and $t_{\mathrm{st}}=10 \mathrm{~mm}$, OF1-II $b_{\mathrm{st}}=100$ $\mathrm{mm}$ and $t_{\mathrm{st}}=10 \mathrm{~mm}$, OF1-III $b_{\mathrm{st}}=200 \mathrm{~mm}$ and $t_{\mathrm{st}}=20 \mathrm{~mm}$, and OF1-IV $b_{\mathrm{st}}=300 \mathrm{~mm}$ and $t_{\mathrm{st}}=30 \mathrm{~mm}$. Except OF1-I (very flexible), the dimensions satisfy the relationship between width and thickness given by Eqn. 9

$$
b_{s t}=12 t_{s t} \sqrt{\frac{f_{y}}{355}}
$$


Fig. 9 shows the relationship between the optimum value of stiffener position $b^{\prime} / b$ and stress gradient $\psi$ for the four groups.

When $\psi \geq 0$, all the curves have enough $h^{\prime} / h$ points in common; when $\psi<0$, the positions of points $h^{\prime} / h$ could be measured in each case. This is explained by the fact that, for $\psi \geq 0$, compression dominates bending, whereas for $\psi<0$, bending dominates compression. In the latter case, stress distributions change in subpanels $b^{\prime}$ and $b^{\prime \prime}$; accordingly, the optimal position differs in each load condition. Increasing the value of $\psi$ in the zone of positive abscissa, the optimal stiffener position moves toward the center-line of the plate, giving $b^{\prime} / h=0.5$ in the case of pure compression stress, $\psi=$ $-1$.

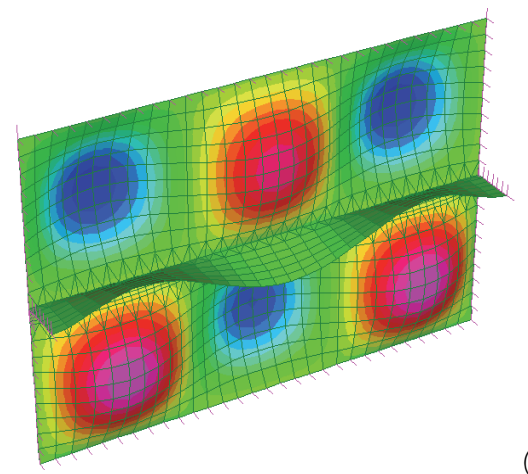

(I)
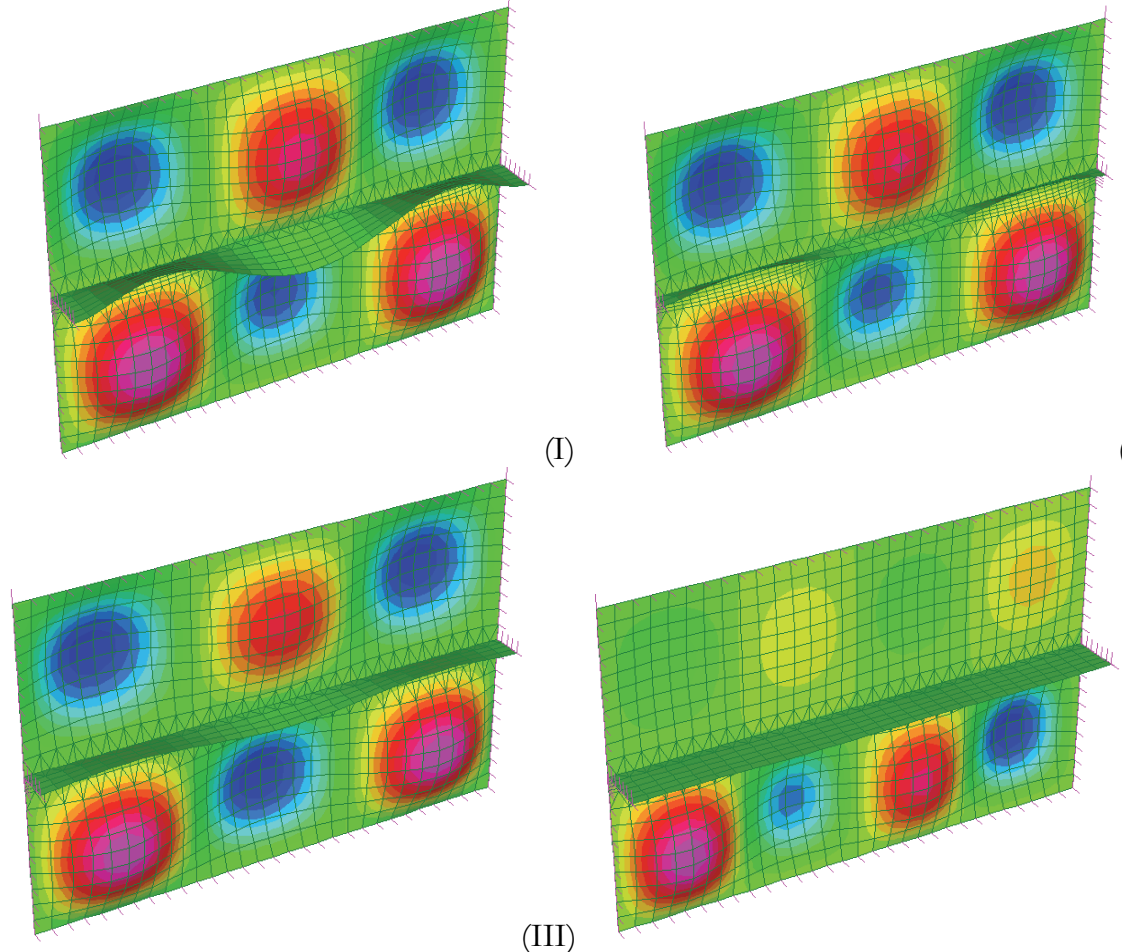

(II)

(IV)

Figure 8: Plate $a=3 \mathrm{~m}, h=1.5 \mathrm{~m}$ and $t=20 \mathrm{~mm}$. Out-of-plane displacements $\Delta z$ from $1^{\text {st }}$ buckling mode.

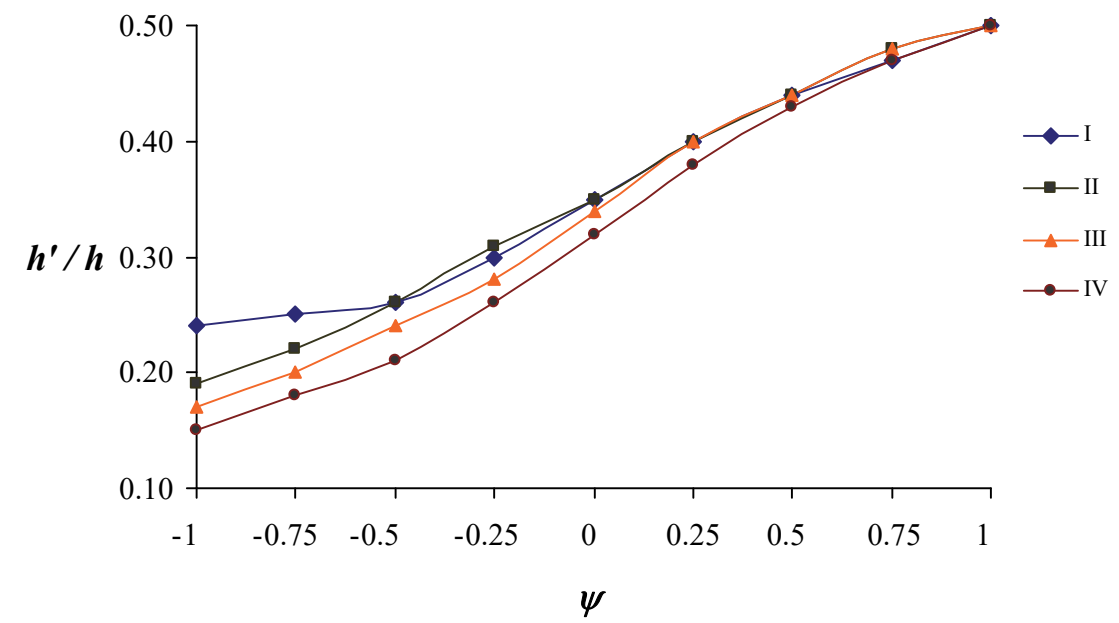

Figure 9: Optimum value of stiffener position $h^{\prime} / b$ vs. stress gradient $\psi$.

Looking at Fig. 8, groups OF1-I and OF1-II show marked deformation of the stiffener after the half-waves produced in the plate plan, reaching critical load; they were therefore classified as flexible types. Group OF1-IV shows a clear distinction 
between the portions of plate divided by the stiffener; there are four half-waves instead of the three found in the other cases. Group OF1-III shows behavior more similar to that of the first two groups, although there is less deformation as the value of the rigid stiffener in OF1-IV is approached. Therefore, an optimal cross-section stiffener in terms of relative flexural stiffness, corresponds to the curve described by group OF1-III and its interpolation equation, Eqn.10, which shows that the optimal position on varying $\psi$ is the following:

$$
h^{\prime} / h=\left(-0.055 \psi^{3}-0.005 \psi^{2}+0.219 \psi+0.341\right)
$$

with coefficient of determination $R^{2}=0.9991$.

Alternately, the linear interpolation equation is:

$$
h^{\prime} / h=(0.179 \psi+0.339)
$$

with coefficient of determination $R^{2}=0.9902$.

Tab. 10 compares the results numerically, according to Eqn.10 and Eq.11, showing a good match.

\begin{tabular}{lccccccccc}
\hline$h^{\prime} / h$ & & \multicolumn{1}{c}{$\psi$} \\
& -1 & -0.75 & -0.5 & -0.25 & 0 & 0.25 & 0.5 & 0.75 & 1 \\
FEM & 0.17 & 0.20 & 0.24 & 0.28 & 0.34 & 0.40 & 0.44 & 0.48 & 0.50 \\
[Eq.10] & 0.17 & 0.20 & 0.24 & 0.29 & 0.34 & 0.39 & 0.44 & 0.48 & 0.50 \\
[Eq.11] & 0.16 & 0.20 & 0.25 & 0.29 & 0.34 & 0.38 & 0.43 & 0.47 & 0.52 \\
\hline
\end{tabular}

Table 10: Results from numerical analyses, Eq.10 and Eq.11.

Fig. 10 shows the curve of the optimum value of stiffener position with respect to stress gradient $\psi$ for optimal crosssection stiffener, in terms of relative flexural stiffness, together with results for the optimum location of stiffener determined according to an energy formulation and a sequential quadratic programming algorithm described in Bedair 1997 [7].

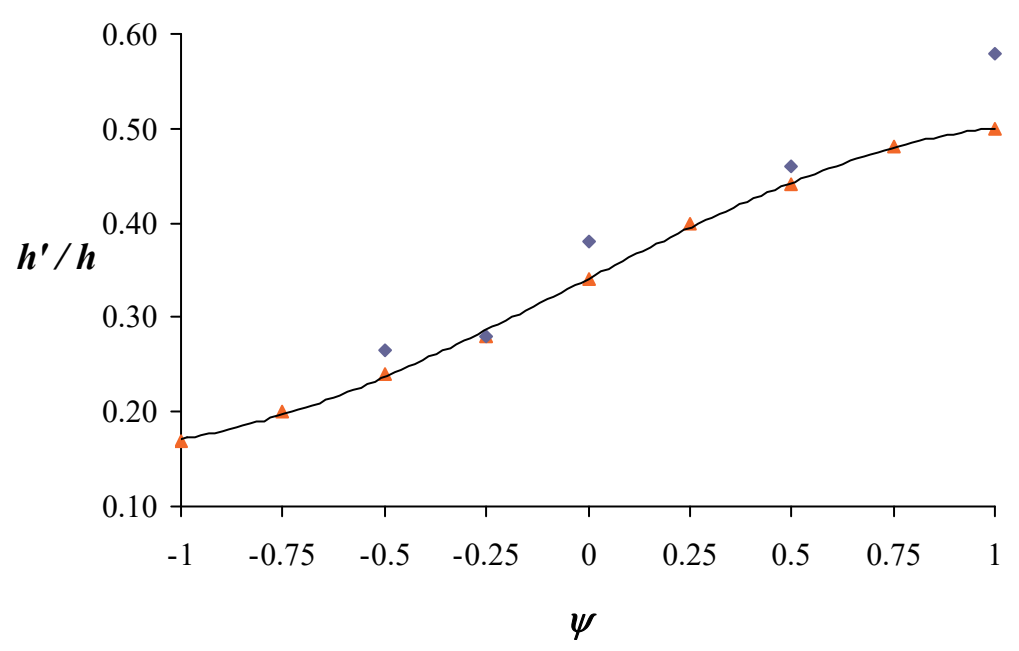

Figure 10: Optimum value of $h^{\prime} / h$ vs. $\psi$ for optimal cross-section stiffener. Comparison between results of Eq.10 and results reported in Bedair, $1997 \bullet$

The comparison shows a good match considering the different approach and schematization of the beam web panel and stiffening. Lastly, the following observations may be derived: for $\psi<0$, the optimal position of the longitudinal stiffener depends on relative stiffness, and flexible and rigid stiffeners can be distinguished, particularly for $\psi=-1$; the best position 
varies from $0.15 \mathrm{~h}$ to $0.25 \mathrm{~h}$, depending on the relative rigidity of the system stiffener / plate (as confirmed in Bedair 1997 [7]). For $\psi \geq 0$, the optimal position is independent of $\gamma_{\mathrm{s}}$ and is a function of $\psi$.

\section{CONCLUSIONS}

7 hree open cross-sections (Flat, T-shaped, L-shaped) and four closed ones (Triangular, Rectangular, Circular, Trapezoidal) longitudinal stiffeners, with equal area and differing second moment of area, were examined. Through linear buckling analysis, buckling coefficient $k$ was used to compare stiffeners contribution in terms of weight per linear meter.

The following conclusions are reached:

- For $\alpha<1.5$, Rectangular cross-section shows the greater value while Circular cross-section appeared to be the best shape to stiffen the plate for $1.5<\alpha \leq 2$.

- Triangular cross-section makes the same contribution to stability with varying $\alpha$.

- Among the open-section stiffeners, the best characteristics were found in the profiles T-shaped and L-shaped crosssections, which show higher second moments of area.

- Flat cross-section makes the lowest contribution to the increase in buckling coefficient $k$.

- Respect to open cross-sections of equal area, with Rectangular, Circular and Trapezoidal cross-sections, $k$ decreases as $\alpha$ increases; local instabilities occur in closed cross-sections with greater slenderness.

- With aspect ratio $\alpha=1.5$ and position of longitudinal stiffener $b^{\prime} / h=0.2$, the minimal value of buckling coefficient $k$ is for Flat cross-section and maximum for Circular.

- Comparing non-stiffened plates with closed cross-section ones, for the range $\alpha<1$, Trapezoidal cross-section is the best.

Finally, longitudinal stiffeners, with optimal flexural stiffness,, set in various positions from the compressed edge were analysed and a useful practical law is given to correlate the best position with respect to variations in stress gradient $\psi$. The results of numerical analyses were compared with those in the literature, to validate the proposed formulation, and a good match was obtained.

\section{REFERENCES}

[1] Timoshenko, S.P. and Woinowsky-Krieger, S. (1959). Theory of plates and shells. Mc-Graw-Hill Book Company.

[2] Xie, M. and Chapman, J.C. (2004). Design of web stiffeners: local panel bending effects. J. Constr. Steel Res., 60(10), pp. 1425-1452. DOI 10.1016/j.jcsr.2004.03.001.

[3] Lee, S.C., Yoo, C.H. and Yoon, D.Y. (2002). Behaviour of intermediate transverse stiffeners attached on web panels. J. Struct. Eng., ASCE, 128(3), pp. 337-345.

[4] Alinia, M.M. (2005). A study into optimization of stiffeners in plates subjected to shear loading. Thin-Walled Struct., 43(5), pp. 845-860. DOI 10.1016/j.tws.2004.10.008.

[5] Alinia, M.M. and Moosavi, S.H. (2009). Stability of longitudinally stiffened web plates under interactive shear and bending forces. Thin-Walled Struct., 47(1), pp. 53-60. DOI 10.1016/j.tws.2008.05.005.

[6] Maiorana, E., Pellegrino, C. and Modena, C. (2011). Influence of longitudinal stiffeners on elastic stability of girder webs. J. Constr. Steel Res., 67, pp. 51-64. DOI 10.1016/j.jcsr.2010.07.005.

[7] Bedair, Osama K. (1997). Influence of stiffener location on the stability of stiffened plates under compression and inplane bending. Int. J. Mech. Sci., 39(1) pp. 33-49. DOI 10.1016/0020-7403(96)00017-3.

[8] Graciano, C. and Casanova, E. (2005). Ultimate strength of longitudinally stiffened I-girder webs subjected to combined patch loading and bending. J. Constr. Steel Res., 61, pp. 93-111. DOI 10.1016/j.jcsr.2004.07.006.

[9] Pavlovčič, L., Detzel, A., Kuhlmann, U. and Beg, D. (2007). Shear strength of longitudinally stiffened panels. Part 1: tests and numerical analysis of imperfections. J. Constr. Steel Res., 63, pp. 337-350.

[10] G+D Computing, (2005). Strand7 User's Manual, Sydney, Australia.

[11] Maiorana, E. and Pellegrino, C. (2011). Linear buckling analysis of welded girder webs with variable thickness. Steel and Composite Struct., 11(6), pp. 505-524. DOI 10.12989/scs.2011.11.6.505. 
[12] Pellegrino, C., Maiorana, E. and Modena, C. (2009). Linear and non-linear behaviour of perforated steel plates with circular and rectangular holes under shear loading. TWS, 47, pp. 607-616. DOI 10.1016/j.tws.2008.11.001.

[13] EN 1993-1-5: 2007. Eurocode 3. Design of Steel Structures. Part 1-5: Stiffened plating subjected to in plane loading. CEN European Committee for Standardization, Brussels, Belgium.

[14] Dubas, P. and Gehri, E. (1986). Behaviour and design of steel plated structures. 1st ed. Institute of Applied Statics and Steel Structures IBS. Swiss Federal Institute of Technology ETH Zurich. ECCS Publication No. 44.

[15] AASHTO (2018). LRFD Bridge Design Specification. Federal Highway Adm. Washington D.C., USA.

[16] EN 1090-2: 2018. Execution of Steel Structures and Aluminium Structures. Part 2: Technical requirements for steel structures. CEN European Committee for Standardization, Brussels, Belgium.

\section{NOMENCLATURE}

a Length of panel

$b_{\text {st }} \quad$ Width of stiffener

D Flexural stiffness of plate

b Height of plate

$h^{\prime} \quad$ Distance between stiffener center and edge subjected to compressive stress

$b^{\prime \prime} \quad$ Distance between stiffener center and edge subjected to tensile stress

$E \quad$ Young's modulus

$I_{\mathrm{st}} \quad$ Second moment of area

k Buckling coefficient

$t$ Thickness of plate

$t_{\text {st }} \quad$ Thickness of stiffener

$x_{2}, z \quad$ Cartesian coordinates

a Aspect ratio

$\gamma_{s} \quad$ Relative flexural stiffness

$\psi \quad$ Stress gradient

$\lambda$ Slenderness of plate

$\sigma_{\mathrm{c}} \quad$ Compression stress

$\sigma_{\mathrm{t}} \quad$ Traction stress

$v \quad$ Poisson's ratio 